\section{Cancer mutations meet drug match}

A study that links tumor genomes with anticancer drugs to support genetic approaches to treatment has produced the world's largest public repository of its kind. The Genomics of Drug Sensitivity in Cancer project has announced that, with its latest data release, it has characterized 1,000 human cancer cell lines; screened them with 453 drugs, both approved and experimental; and identified close to half a million genome-drug associations. The data in this carefully curated dataset, which are free to access on the open source website, have proven to be a valuable resource for the research community and a formidable resource towards improving patient response during cancer treatment by enabling personalized clinical decisions. The genomic variants could also prove to be biomarkers for response to drugs. Funding for the project is from the UK-based Wellcome Trust, and the work was done by researchers at the Wellcome Sanger Institute in Cambridge and integrated with Boston's Broad Institute's Cancer Dependency Map project. The website has 350 users logging on each day, and project leaders say the dataset has supported more than 70 research studies, including the discovery of PARP inhibitors in childhood bone cancer.

Published online: 4 September 2019 https://doi.org/10.1038/s41587-019-0258-0

\footnotetext{
66 hat makes this unscrupulous action even more appalling is the fact that AveXis was the beneficiary of numerous federal taxpayerfunded benefits and incentives, including obtaining Fast Track, Breakthrough Therapy, and Priority Review designations - ensuring that Zolgensma would be sped through the regulatory approval process." $A$ group of US senators writes about Novartis's failure to inform the FDA that some data were falsified until after the drug was approved. (STAT, 9 August 2019)
}

“Because you can start cheaply, it's now possible to start a biotech company the way people start a tech company." Blogger Jared Friedman argues that new infrastructure is bringing down the cost of starting a biotech. (Y Combinator, 5 August 2019)

\footnotetext{
“You can't just sprinkle drugs into food, and that's what you would be doing if you put CBD [cannabidiol] wildly into the food supply." Former FDA Commissioner Scott Gottlieb says the FDA should have more, not less, authority to regulate CBD in food. (Washington Journal, 7 August, 2019)
}

and to a single organ in a median $63 \%$ of patients. CancerSEEK's median sensitivity was $43 \%$ for stage I cancers, $73 \%$ for stage II cancers and $78 \%$ for stage III cancers. In the absence of any screening tests for most types of cancer, that level of sensitivity may be sufficiently useful for detecting cancer earlier than is now the case. "It's important we say 'earlier' as opposed to 'early"' Kafka says. "It isn't just a function of finding cancer at stage I, although it would be great if we could do that."

Thrive is now collaborating with Johns Hopkins and the healthcare provider Geisinger on a five-year prospective screening study, DETECT, which has enrolled 10,000 healthy female participants ages 65-75. An interim data read-out, based on one year of follow-up, is expected in the middle of next year.

Freenome is casting the net more widely than its rivals in the hunt for early signs of cancer. Its 'multi-omic' approach looks at cell-free DNA, methylation signatures and proteins, both from nascent tumors and from the immune system. It then integrates these signals using artificial-intelligencedriven analytics. The foundation for Freenome's approach is that circulating cells of the immune system are detecting and responding to cancer in its earliest stages; these are ignored by most other companies, which focus their liquid biopsies on the ctDNA fraction shed exclusively by tumors. "They ignore the rest of the 99-plus percent of the cell-free DNA," says chief scientific officer Jimmy Lin. In one publicly disclosed retrospective study, Freenome obtained 85\% mean sensitivity (at $85 \%$ specificity) for stage I and stage II colorectal cancers testing cell-free DNA from tumor and immune cells, without including any other analytes. "We believe we can well exceed that," says Freenome cofounder and chief partnerships officer Charles Roberts.

The multimodal approach is "valid," says Eleftherios Diamandis, head of clinical biochemistry at Mount Sinai Hospital and University Health Network in Toronto and at the University of Toronto, but he adds a caveat: "As you increase the number of analytes you measure, you increase the sensitivity-you detect more cancers-but you may decrease the specificity at the same time," he says. Freenome's Lin counters that its proprietary machine-learning approach to interpreting the data its test generate can avoid this problem.

Freenome is taking a stepwise approach to develop its platform, with an initial focus on colorectal cancer. The choice of indication reflects the fact that colorectal cancer already has a well-defined screening test, Exact Sciences' Cologuard stool DNA test, against which Freenome can pit its own test. A validation study called AI-Emerge, which involves 3,400 participants, including healthy volunteers undergoing routine colonoscopy and people with newly diagnosed colorectal cancer, is underway and will read out early next year. Freenome is not alone in the colorectal cancer space. Guardant Health, an early leader in liquid biopsy testing for treatment selection, is also developing an early detection test for colorectal cancer, Lunar-2, which combines sequence and epigenomic analyses of ctDNA, as well as a bioinformatics classification system for eliminating benign clonal mutations arising during hematopoiesis. "Later in the second half of 2019, we're going to start our pivotal prospective screening study in colorectal cancer," says AmirAli Talasaz, Guardant's cofounder, president and chief operating officer. This will recruit 10,000 participants with average risk of developing colorectal cancer.

At present, population-based cancer screening is very much a hit-and-miss affair. Computed tomography, colonoscopy, Pap testing and mammography have demonstrated varying degrees of benefit in reducing deaths from lung cancer, colorectal cancer, cervical cancer and breast cancer, respectively, but the continued mortality from those conditions underlines the deficiencies of these methods. For many other types of cancer, including pancreatic cancer and liver cancer, there are no effective screening tests. Liquid biopsy developers have plenty of scope, therefore, to bring a whole new approach to cancer screening. But developing an understanding of the clinical significance of a test result will be every bit as important as developing an accurate test. "We know already we can detect cancer early," says Nitzan Rosenfeld, cofounder and chief scientific officer at Cambridge, UK-based Inivata, pointing to the identification of maternal cancers during cell freeDNA-based non-invasive prenatal testing for aneuploidy (abnormal numbers of chromosomes) during pregnancy. "The hard part is not whether it's possible, but whether it's clinically useful or not," he says. Diamandis concurs: "Screening may lead to overdiagnosis, which may lead to overtreatment," he says. But, Stebbing notes, managing this problem is an intrinsic aspect of cancer care. "We have problems with overdiagnosis already, with mammography." Underdiagnosis of early-stage cancers is still a much bigger problem.

\section{Cormac Sheridan \\ Dublin, Ireland}

Published online: 6 August 2019

https://doi.org/10.1038/d41587-019-00022-7 